\title{
Keyword index to Volume 85
}

Accelerated chemotherapy 1444 Acquired resistance 1064 ADCC 463

Adenocarcinomas 1023, 1153, 1371, 1781

Adenomatous polyposis coli 69,98

Adjuvant chemotherapy 827 , 1251

Adjuvant therapy 1437

Advanced breast cancer 141

Advanced gastrointestinal cancer 1265

Aetiology 1667

Ageing 930

Aggressive fibromatosis 98

AIDS 1298

Alcohol 46, 341, 678

Alcohol intake 1700

ALIMTA 649

Allelic imbalance 1201

Alphafetoprotein 1119

Aminolevulinic acid 279, 727

Aminolevulinic acid derivatives 279

Aminolevulinic acid hexylester 727

5-Aminolevulinic acid 1572, 1794

5-Aminolevulinic acid esters 1572,1794

Aminopeptidase N 924

Amsacrine 747

Androgen receptors 36, 1928

Androgen regulated transcripts 393

Angiogenesis 266, 439, 473, 863, 881, 1396, 2010

Angiogenesis inhibition 1540

Angiotensin 1396

Angiotensin II 1640

Anthracycline-containing regimen 141

Anthracycline/taxane resistance 798

Antiangiogenic therapy 584

Anti-apoptotic genes 405

Anti-cancer drugs 242

Antidotal activity 1964

Antimicrotubule agents 735

Antineoplastic drugs and medications 1400, 1611

Anti-p53 antibodies 1883

Antisense 1753

Anti-tumour activity 741

Apoptosis 115, 273, 279, 303, 453, 584, 764, 898, 902, 1047, 1403, 1522, 1564, 1764, 1771,
1781, 1801, 1914, 1964, 2004, 2017

AQ4N + cisplatin 625

Archival smears 398

Arimidex 317

Aromatase inhibitors 317

Asparagine synthetase 930

Assam 661

Asthma 1317

ATP 1987

Attenuated adenomatosis polyposis coli 523

Avascular necrosis 1624

Axillary surgery 668

5-Aza-2'-deoxycytidine 1351

Basal cells 422

Basal cell carcinomas 683, 1883

Basic fibroblast growth factor 1706

BAX 1359

Bax 1557

BCL-2 1359

Bcl-2 115, 273, 1557, 1564, 1764, 1781

Bcl-2 family 1801

bcl-2 phosphorylation 1403

Bcl-X 1557

Benign prostatic

hyperplasia 576

Betel nut chewing 658, 661

BH3 115

Biochemotherapy 1871

Biomarkers 1952

Bioreductive drugs 1137

Birthweight 428

Bispecific antibodies 152

Bladder cancers 568 ,

1137, 1412, 1515, 1887

Bladder neoplasms 552, 977

Blood flow 1640, 1655

Bloom's syndrome gene product 261

Body height 959

Body mass 1680

Body mass index 372

Body surface area

estimation 23

Body weight 346

Body weight/body mass index 984

Bone resorption 78

Book reviews 1609, 1810, 1811

BQ123 1759

Brain tumours 285, 1396

BRCA1 538

BRCA1 36, 845, 850,

1201,1675
BRCA1 gene 1347

BRCA2 36, 850, 1201, 1675

Breast cancer $1,69,78,85,171$,

$141,225,317,372,422,428$,

490, 538, 618, 637, 668, 798,

$859,869,917,959,962,1014$,

$1102,1157,1240,1280,1289$,

1340, 1522, 1675, 1680, 1731,

1764 1878, 1914, 1958, 2017

Breast cancer cells 909

Breast cancer cell lines 902

Breast cancer prevention 1838

Breast cancer risks 36

Breast cancer therapy 147

Breast conservation therapy 668

Breast neoplasms 362, 1347

Breastfeeding 1685

Bromocriptine 1611

$\mathrm{C} 225584$

c-KIT/SCF 405

Cachexia 297

Calman-Hine report 166

Camptothecin resistance 1077

Canada 1335

Cancer chemoprevention 618

Cancer chemotherapy 1219

Cancer gene therapy 1592

Cancer genetics services 166

Cancer genomics 1492

Cancer immunotherapy 1527

Cancer immunotherapy,

human 1722

Cancer incidence 1332

Cancer patients 641

Cancer registry 367,1251

Cancer screening 1332

Cancer vaccine 713

Cancer-testis antigens 713

Carbonyl reductase 1032

Carboxymethylbenzylamide dextran derivative 917

Carcinoembryonic antigens 1787

Carcinogenesis 1922

Carcinomas in situ 213

Carotenoids 977

Case-control studies 337, 341, 398, 683, 966, 991, 1326, 1667

Caspase 31522

Catechol oestrogens 859

Catechol- $O$ methyltransferase 859

$\beta$-Catenin 64, 98

$\gamma$-Catenin 64

Cathepsin S 1193

Caucasians 171
CCR1 891

CCRF-CEM 1400

CD4+ T cell clones 1527

CD44(var) 924

CDC25A 412

CDC25B 412

CDDP-based chemotherapy 1064

CDHP 939

$C D K 4$ genes 836

CDKN2A 527

$C D K N 2 A$ genes 836

CDKN2A gene 1383

cDNA 1351

cDNA array 538

Cell cultures 285

Cell cycles 1771

Cell cycle arrest 898

Cell growth 1978

Cell lines 1014, 1492

Cell mediated immunity 473

Cell proliferation 1771

Cell scattering 1412

Cervical cancer 93, 398, 791, 966, 1153, 1675

CHART 1113

Chemokines 891

Chemopotentiation 625

Chemosensitivity 1425

Chemotherapy $649,808,816$, 1099, 1240, 1444, 1456, 1646, 1850

Chemotherapy steroids 1624

Child development 1611

Childhood cancer 337, 350, 1680,1685

Chk2 293

CHK2 mutations 209

Chlorambucil 764

Chlorpromazine 1998

chLym-1 463

Chondrosarcomas 176

Chromosomal aberrations 1900

Chromosomal

radiosensitivity 1157

Chromosome 10p15 1510

Chromosome 11 531, 1894

Chromosome 17 705, 1894

Chromosome aberrations 293

Chromosome instability 1887

Circulating tumour cells 431

Cisplatin 9, 823, 1124, 1206, 1456

Cleavable complex 1585

Cleavase fragment length polymorphism 845

Clinical features 538

Clinical resistance 509

Clinical trials 1432, 1467 
Clonal evolution 182

Clonogenic assays 1585

CMF 1106

CNS toxicity 1233

Co-activators 1928

Co-localisation 909

Cohort studies 346, 362

Colchicine 1998

Collagenase 383

Colon cancer 772, 1251, 1437 , 1572

Colon neoplasms 1047

Colonoscopy 972

Colorectal cancer 504, 523, 641, 692, 787, 827, 972, 1037, 1258, 1486, 1695, 1759, 1771, 1937

Colorectal liver metastases 1640, 1759

Colorectal neoplasms 346, 357

Colorectal tumours 764, 1492

Comparative genomic

hybridization 213,422 697, 1492

Competing risks 1113

Computer touchscreens 1842

COMT 859

Consanguinity 1675

Consultations 1273

Continuous

hyperfractionated

accelerated radiotherapy 1113

Contralateral breast cancer 850

CpG-methylation 1168

Cremophor EL 1124, 1472

CTL epitope peptides 1722

${ }^{62} \mathrm{Cu}$-PTSM 1640

Cyclin-dependent kinase inhibitors 1515

Cyclo-oxygenase-2 1023

Cyclophosphamide 798

Cytochrome P450 242

Cytoreduction 1824

Cytosols 85

Dacarbazine 1467

DC101 584

DCC 199

Death associated protein

kinase family 1801

Decorin 228

Deletion mutations 176

Denaturing gradient gel

electrophoresis 235, 523

Dermatomyositis 41

Dexamethasone 1099

Diagnosis 225, 656

Diarylsulfonylureas 1400

Diet 341

Dietary cholesterol 357

Differential displays 1162

Differentially expressed genes 393

Differentiated thyroid cancer 875

Differentiation 590, 1944, 2004

Diltiazem 1577

Dimethylbenz $[a]$ anthracene 428
Dlk/ZIP kinase 1801

DNA damage 898

DNA methylatioin 1887

DNA microarrays 107

DNA ploidy 182

Docetaxel 242, 1247

Dorsal root ganglia 1219

Down-regulation 220

Doxorubicin 1964, 1987

Drug combinations 1077

Drug resistance 735,902 ,

1175,1425

Drug targeting 747

Ductal carcinoma 225, 422

dUTP 446

dUTPase 446

Dysplastic nevi 1304

Early detection 803

Early onset 859

EBAG9/RCAS1 1731

E-cadherin 1958

Efficacy 1326

EGFRvIII 1211

Eggs 357

Egypt 1037

ELISA 1193

ELISPOT 1738

Emetogenic chemotherapy 1099

EMT 1412

Endometrial cancer 333, 546

Endoscopy 972

Endothelial cells 584

Endothlin-1 1759

Enzyme-linked immunosorbent assay 1193

EO9 1137

EORTC QLQ-C30 1265

Epidemiology 962

Epidermal growth factor 303, 84

Epidermal growth factor receptors 1600

Epidermal growth factor receptor antagonists 1600

Epidermal growth factor receptor ligands 1412

Epirubicin 1106, 1456

Epithelial ovarian cancer 1032

Epitopes 875

Epstein-Barr virus 350, 997

ErbB2 705, 1787

ET $_{\mathrm{A}}$ antagonists 1759

$\mathrm{ET}_{\mathrm{B}}$ antagonists 1759

Ethnicity 350

Etoposides 747

EUROCARE 787

Ewing's tumour 1646

Excess risk 362

Exercise 962

Exostoses 2176

Expression 1162, 1211

Extracellular matrix 1968

Extracellular signalregulated protein kinase-2 1403

Extraintestinal mucosa-

associated lymphoid tissue lymphoma 1462

Extravasation 1387

Failure-specific prognostic factors 1113

Falkland Islands 1332

False negative cytology 398

Familial colorectal cancer 523

Familial melanoma 836

Familial papillary thyroid carcinoma 1831

Family history 674

Fas-L 1185

Fats 357

Fc $\gamma \mathrm{R} 463$

Females 341

Fertility patterns 362

Fibroblasts 293

Fibroblast growth factor-8 576

Fine needle aspiration 1102

FISH 1201

Flexible modelling 795

Fluorescein-colchicine 1998

Fluorescence 1572

Fluorescence in situ

hybridization 697, 1201

Fluoropyrimidine 439

5-Fluorouracil 798, 1258, 1937

Focal adhesion kinases 228

Folate 977

Follicular lymphomas 29

Follow-up studies 997

Founders 527

Fractionated administration 9

Fragile histidine triad genes 247

France 1664

Fusion transcripts 1535

$\alpha$-GalCer 741

Gallbladder cancer 1922

GalNAc glycoproteins 1014

Gangliosides 285

$\mathrm{G}_{1}$-arrest 293

Gastric adenoma 1147

Gastric cancer 199, 1147 , $1317,1322,1585$

Gastrointestinal cancer 1265

Gastro-oesophageal reflux 1317

Genes 1211

Gene amplification and expression 518

Gene clones 1162

Gene expression 107, 266, 1206 , 1372

Gene mutations 64

Gene overexpression in cancer 190

Gene therapy 1432

Genetics 1499

Genetic analysis 687

Genetic counselling 166

Genetic polymorphism 859

Genistein 618

Germ cell tumours 823, 1119

Germ line mutations 523, 836

Glioblastoma 518

Gliomas 55, 1185, 1396
Glomerular filtration rates 325

Glucocorticoids 683, 1611

GM-CSF 152, 1444

Good practices 1251

gp 100/pmel17 1738

$\mathrm{G}_{2}$-phase 1157

G-protein expression 758

Granisetron 1099

Granulocyte-macrophage colony-stimulating factor 1467

Growth hormone antagonists 428

Growth inhibition 453

Growth kinetics 490

Gynaecological oncologists 1824

Haemangioma 362

Haematolymphoproliferative disorders 997

Haemophilus influenzae type b 337

Head and neck cancer 630, 1055

Head and neck squamous cell carcinoma 649

Health service organisation 166

Health services accessibility 497

Height 1680

Helicobacter pylori 1322

Helix pomatia lectin 1014

Helper T cells 1738

Heparan sulfate 1094

Hepatocarcinogenesis 697

Hepatocellular carcinoma

228, 697, 1162, 1850

HER2 152

HER-2/neu 176

HER-2/neu peptide 1527

Hereditary breast cancer 209,845

Hereditary multiple exostoses 176

Hereditary non-polyposis colorectal cancer 1486

Herpesvirus 8, human 379

Heterogeneity 182

High throughput screening 1206

High-dose chemotherapy 484, 1240

High-dose oestrogens 147

Histology 1347

HIV 1298

hMLH1 1147

hMSH2 1147

Hodgkin's disease 350

Homing 1387

Hormone replacement therapy 674

HPA 1014

HPMA copolymer 1070

HPV-infection 1153

hTERT 752

hTR 1510

hTRF1 752

Humans 171, 1372, 1700

Human herpesvirus 81298

Human papillomavirus 966, 1551

Humoral immunity 473 
Hyaluronan receptors 600

Hyaluronan synthase 600

Hypermethylated in cancer gene-1 1878

Hypermethylation 69, 930, 1878

Hypersensitivity pneumonitis 1247

Hypomethylation 563

Hypoxia 891, 1577

Hypoxia inducible factors 881

I1307K APC mutation 1368

IgG uptake 1968

IgGK Fab 875

Immune evasion 1047

Immunocytochemistry staining 1713

Immunohistochemistry 576, 1193, 1731, 1922, 1937

Immunoluminescence 924

Immunophototherapy 1787

Immunosuppression 997

Immunosuppressive therapy 683

Immunotherapy 152, 713

In situ hybridization 383, 869,1510

Incidence 1322, 1335

Information and support requests 641

Inherited predisposition to cancer 1368

Initial therapies 29

Insulin 346

Insulin-like growth factor binding protein-3 74, 1695

Insulin-like growth factor system 147,1838

Insulin-like growth factor-1 $74,428,991,1695$

Insulin-like growth factor1R inhibitors 2017

Insulin-like growth factor-2 1695

Intercellular adhesion molecule-1 924, 135

Interferon 29

Interferon- $\alpha 1130$

Interferon- $\alpha$ sensitivity 107

Interferon- $\gamma 741$

Interleukin-1 receptor antagonists 1600

Interleukin-2 1130

Interleukin-6 518

Inter-observer variability 1634 Interstitial fluid pressure 1968 Intraperitoneal topotecan 1627 Intrauterine exposures 959 Intravital microscopy 431 Invasion 122, 869, 1600 Ionizing radiation 293, 362

Irinotecan 9

Irradiation 1055

Isolated limb infusions 157

Isotretinoin 630

Isotypes 735

Italy 379

JNK 303

Kallikrein 220

Kaposi's sarcoma 379, 1298

Kaposi's sarcoma herpesvirus

1298

Keratin 14422

Keratinocytes 630

Ki-67 1106, 1781

Kidney neoplasms 984

Ki-ras 692

Kirsten ras mutations 692

KLK10 220

Knockout mouse 431

Laminin-1 1387

Laminin-5 383

Langerhans cells 1944

Laryngeal neoplasms 678

Laser capture microdissection 235

Letters to the Editors 137, 139 , 140, 780, 781, 1418, 1606 1607

Leucaphereses 1713

Leucovorin 798

Leukaemia 337

Leuprolide 333

Li-Fraumeni syndrome 293

Li-Fraumeni-like syndrome 209

LINE-1 1887

Lipid-mobilizing factor 758

Lipolysis 758

Liposomal doxorubicin 1850

Liver 1540

Liver metastases 504, 612

Liver microcirculation 431

Lombardia 379

Losartan 1396

Loss of heterozygosity

531, 1201, 1351, 1510, 1894

Loss of heterozygosity, 1p36 204

Low-incidence areas 721

67LR 1387

Lugol 1006

Lung cancer 235, 255, 1326

Lung neoplasms 678

Lung tumours 1193

Luteinizing-hormone

releasing hormone

analogues 333

LY231514 649

Lycobetaine 1585

Lymph node metastases 93, 255, 1032

Lymphatic mapping 791

Lymphocytes 1731

Lymphocytic thyroiditis 1831

Lymphoma 463, 930, 1462

Lymphopenia 816

Lymphoscintigraphy 791

Macrophages 285

MAGE 713

MAGE-A3 1340

Magnetic resonance imaging 1655
Maintenance 29

Major histocompatibility complex-II 1193

Malignancy 1611

Malignant cell growth 618

Malignant melanomas 1871

Malignant mesothelioma 600 , 863

Mammary carcinoma 546

Mammary glands 1522

Mammographies 225

Management 808

Mantova 379

Mapping 1510

Marimastat 1865

Mastectomy 668, 1952

Material deprivation 350

Matrigel 590

Matrix metalloproteinases 1865

Matrix metalloproteinase-1 1600

Matrix metalloproteinase-2 1706

Matrix metalloproteinase- 9 1706

Maturation 1944

MBD2 1168

MCF-7ras 917

MDA-MB-231 1978

MDM2 721, 1746

MDR 1987

MDR-1 1175

$m d r-11746$

Meat 357

MeCP2 1168

Mediterranean populations 1304

Medroxyprogesterone acetate 1

Medullary thyroid

carcinoma 1546

Medulloblastoma 705, 1801

Melanomas 107, 157, 527, 741, 803, 1304, 1467, 1738

Melanoma, familial 836

Melanoma, human 1944

Melanoma thickness 1304

Melphalan 157

Men 641

Menopause 346

Meta-analysis 984, 991, 1700

Metabolism 630

Metalloproteinases 55, 122

Metastases 78, 285, 608, 1014 1937

Metastatic germ cell tumours 823

Metastatic lesions 313

Metastatic non-small-cell lung cancer 1452

Methodology 1655

Methylation 199

Methylation-specific polymerase chain reaction 69

MHC alleles 1527

Microdialysis 157

Micrometastases 490, 1340
Microsatellite instability

568, 1037, 1064, 1147, 1486

Microsimulation models 1280

Migration 1387

Minisatellite 1486

Mismatch repairs 568

Mitochondria 1801

Mitogen-activated protein

kinase 303, 1175, 1403

Mitomycin C 1137

MN/CA9 563

Molecular and cellular mechanisms 1233

Molecular diagnostics 1546

Molecular profiles 538

Molecular-based diagnosis 102

Monoclonal antibodies 875 , 1193, 1787, 1964

Monofunctional ZD2767 analogues 764

Monozygotic twins 493

Morbidity 325

Morphology 1535, 1978

Mortality 803, 1664

Mouse 930

MRP1 1564

MSH3 568

MSH6 568

Mucositis 1964

Multicell tumour spheroids 727

Multidrug resistance 1998

Multiple myeloma 1387

Multitargeted antifolate 649

Multivariate analysis 509

Muscle proteolysis 297

Mutational analysis 845

Mutations 199, 1383

MVR-PCR 1486

MYC 705

MYCN 531

Myeloma 325

Myoepithelial 422

NADH oxidase 1400

Neoplasms 261, 1700

Neuraminic acid 285

Neuritic differentiation 1564

Neuroblastomas 182, 493 ,

531, 1564, 2004

Neurotoxicity 1219

Neutrophils 463

Newborns 1295

Nipple aspirate fluid 1952

Node-negative primary breast cancer 795

Non-astrocytic tumours 204

Non-Hodgkin's lymphoma 1298, 1425, 1900

Non-melanoma skin cancer 683

Non-platinum-based regimen 1452

Non-seminomatous germ cell

tumours 608

Non-small-cell lung cancer

9, 14, 129, 881, 939

1113, 1247, 1456, 1706

Normal cytology 398 
Normal epithelial cellspecific 1 genes 220

Norway 959

Nottingham prognostic index 795

NQ01 1137

Nucleolus 1219

Nude rat 2004

Nurse-led clinics 1853

Nutrition 959

Obesity 984

Odds ratio 991

Oesophageal cancer 341 , 658, 661, 721, 1006, 1317, $1499,1667,1671,178$

Oestradiol 317

Oestrogen 317, 1731

Oestrogen receptors 372,546

Older women 1289

Oligodeoxynucleotides 1753

Oncogenes 697

Oncologists 1634

Oncology 1842

Ondansetron 1099

Oral administration 1472

Oral cancer 46

Oral cavity neoplasms 678

Oral squamous cell

carcinoma 122

Oral topotecan 1124

Orthotopic xenografts 608

Osteoclasts 78

Osteosarcoma 1968

Outcome 325

Ovarian adenocarcinoma 1351

Ovarian cancer 190, 313 , 687, 891, 944, 1064,

1124, 1175, 1359, 1540,

$1592,1627,1753,1824$

Ovarian tumours 247

Overall survival 944

Oxaliplatin 1258

P15 1515

p16 gene 1383

p21 1359

1 p36 204

p42/p44 kinases 1978

p53 293, 1102, 1153,

$1499,1764,1781$

p53 898, 902, 1878

p53 protein 1937

p53 tumour suppressor 1813

P63 721

p73 1499, 1551

p73 gene 204

Paclitaxel 141, 303, 902, 1472

Paediatric oncology 23

Pain 497

Pain measurements 497

Palliation 1265

Palliative care 816,1478

Pancreatic cancer 612, 1865

Pancreatic cancer cells 752

Papillomavirus, human 398

Parathyroid-hormone- related protein 1722

Parotid glands 1055

Past use 674

Patients 1634

Patient participation 1273

Patient prognosis 313

Patterns of care 1251

Pemetrexed disodium 649

Pellet extracts 85

Pentoxifylline 1577

Pepsinogen C 228

Peptides 115

Performance status 1634

Peripheral benzodiazepine

receptors 1771

Peripheral blood stem cells 1119

Peru 966

P-glycoprotein 1746, 1998

Pharmacogenetics 1425

Pharmacogenomics 107

Pharmacokinetics 1627

Phase I studies 1627

Phase II studies 9

Phenotypes 1368

Photodynamic therapies 279, 1794

Phthalocyanine 1787

Physical activity 962, 1311

PI-3K 303

Pigmentation 1304

Plasma membrane 1400

Plasminogen binding 909

Platelet-derived growth factor 1706

Platinum drugs 1219

Ploidy 14

Pluronic block copolymers 1987

Po Valley 379

Poisson regression 1335

Poliovirus vaccines 1295

Poly(ADP)ribose polymerase 1403

Polymer prodrugs 1070

Polymer-directed enzyme prodrug therapy 1070

Polymer-enzyme conjugates 1070

Polymerase chain reaction 1546

Polymorphisms 36, 171, $827,1153,1499,1504$

Polymyositis 41

Population health impacts 1280

Population studies 1347

Positron emission tomography 1640

Preclinical pharmacology 484

Prediction of response 1871

Predictive factors 1478

Pre-mRNA splicing 190

Present use 674

Prevention 428, 1280

Primary brain lymphomas 204

Primary breast cancer 74,85 , 1958

Profiles 1211

Progesterone receptors

372,546
Prognosis $14,85,313$ $367,412,552,705,808$, $831,863,881,1032$ $1359,1535,1557,1878$

Prognostic factors 1646, 1671

Prognostic indicators 1958

Prognostic models 944

Prognostic values 1634

Progression 869

Proliferation 772

Proliferation status 727

Prophylactic treatment 1055

Prostaglandins 1023

Prostate androgen regulated transcript 1393

Prostate cancer 115,152 , $393,453,557,576,64$ 656, 991, 1504, 1722, 1928

Prostate spheroids 590

Prostate-specific antigens 656

Prostate-specific antigen mRNA bearing cells 557

Prostatic neoplasms 497

Protease-activated receptor-2 772

Proteins 357

Proteoglycans 1094

Proteolysis 74

Proteolysis-inducing factor 297

Protoporphyrin IX 727, 1572

Protracted venous infusion 1258

PR-transfection 1978

P-selectin 431

Psychological distress 1842

Quality of life 497, 1265, 1478

Quantitative reverse transcriptase polymerase chain reaction 393

RAC 31928

Radiation 1611, 1853

Radiosensitivity 1577 . 2010, 2017

Radiotherapy 1233, 1671

Raf 1753

Ras oncogenes 235

RASCAL II Study 692

RCAS1 1922

Real-time quantitative reverse transcriptionpolymerase chain reaction 102

Receptors 171, 584, 1023

Receptor subtypes 266

Recombinant adenoassociated virus 1592

Recombinant adenovirus 1592

Record linkages 41

Rectal cancer 1437

Regional chemotherapy 157

Regional intra-arterial treatment 504

Regional variations in survival 637

Registries 497
relA 1914

Renal cancer 1130

Renal cell carcinoma 563 , 924, 953, 1372

Resistance 1746

Ret oncogene activation 1831

Retinoblastoma protein family 546

Retinoic acid 122, 2004

Retinoic acid receptors 453

Retinoic acid receptor antagonists 453

Retinoids 630

Reverse transcriptionpolymerase

chainreaction 55,405 ,

557, 1119, 1546, 1713

Rhabdomyosarcoma 831, 1746

Ribozymes 1185

Risk factors $428,816,972$ 984, 1304, 1499, 1700

Rituximab 1619

RNA polymerase 190

Routine practices 1842

RT-PCR 1340

RU486 morphology 1978

S-1 939

Salvage chemotherapy

509, 798

Schistosomiasis 1037

SCID 608

Scintigraphy 1462

Screening 656, 1289, 1326, 1842

Second malignancies 997

Second-line chemotherapy 1456

Selective oestrogen receptor modulators 1838

Sensitisation 1987

Sentinel lymph nodes 791 , 1340

Sequential chemotherapy 14

Serine protease 772

Serine/arginine-rich

proteins 190

Serology 337

Serous carcinoma 247

Sexual habits 46

Sialic acid 285

Side-effects 1853

Sigmoidoscopy 972

Signal transduction 618

Skin tumours 1883

Small-cell lung cancer $808,1444,1713$

Smoking 341

Sodium phenylacetate 917

Solid tumours 484

Soluble Fas ligands 1047

Somatostatin 266, 1462

Somatostatin receptors 266

Soya 618

Soyfood 372

Splicing factors 190

Sporadic renal cell carcinoma 1226

Squamous-cell cancer of

the head and neck 1383 
Squamous-cell carcinoma $630,658,683,721$, 1006, 1883

Squamous cell carcinoma of the oesophagus 412

Squamous papillomas 1794

SR family 190

SR-A1 190

Stages 787

Staging 863

Superantigens 129

Supportive care 1853

Suppression subtractive hybridization 228, 1206

Surgery 1824

Surgery signals 490

Survival 367, 576, 637, $787,808,1251,1492$ 1850, 1900

Survival outcomes 1928

Survival rates 1671

Susceptibility 1504

SV40 1295

Synergism 917

Synovial sarcoma 405, 1535

Tamoxifen 317, 1106, 1130, 1280

Taxol 1175

tcf/lef transcription factors 98

Tegafur 939

Telomerase 752, 1006

Telomerase repressor genes 1510

Telomere 898

Telomeric restriction

fragment 752

Tenascin-C 383
Testicular cancer 213, 220, 1624

Testicular germ cell tumours 608

Thalidomide 953

Thymidine phosphorylase surexpression 439

Thymidylate synthase 446 , 827, 1937

Thyroglobulin 102

Thyroid cancer $102,875,1335$

Thyroid carcinoma, familial papillary 183

Thyroid peroxidase 875

Time trends 1335

Tissue inhibitors of metalloproteinases 55

Tissue-type plasminogen activators 122

Tobacco 46

Tobacco chewing 661

Topoisomerase IIa 747

Topoisomerase IIb 747, 1585

Topoisomerase inhibitors 1077

Topotecan 1627

Toxicity 1850,1865

TP53 721, 1359

Transfection 735

Transforming growth factor- $\beta$ 687

Transforming growth factor- $\beta_{1}$ 612

Transitional cell carcinoma, human 1515

Transitional cell

carcinomas of the urinary

bladder 1894

Translocation 831
Treatment-related deaths 816

Trends 1664

TRF1 898

TRF2 898

Trypsin 772

TSC1 1226

TSC2 1226

Tubulin 735

Tumorigenesis 1185

Tumorigenicity 1914

Tumours 1655

Tumour angiogenesis 439

Tumour cell contamination 1713

Tumour dormancy 490

Tumour growth control 2010

Tumour incidence 674

Tumour markers 393, 552

Tumour perfusion 1577

Tumour spreading 600

Tumour suppressor genes 220 , 697, 1887

Tumour targeting 1070

Tumour therapy 1540

Tumour-infiltrating lymphocytes 1722, 1871

Tumour-rejection antigens 713

Tumoural markers 1883

Tyrosinase 1738

Ubiquitin-proteasome proteolysis 297

Ultraviolet radiation 1504

Ungeremine 1585

uPARs 85, 924

Uracil misincorporation 446

Urokinase 909

Urokinase-type plasminogen activators 85,924

Urologic neoplasms 977

Uterus 1023

V126D 527

Vaccines 1527, 1738

Vascular endothelial growth

factor $273,313,576$, $584,612,795,1706$

Vascular endothelial growth factor receptors 255

Vascular endothelial growth factor-C 93, 255

Vascular endothelial growth factor-dependent

proliferation 2010

Vascular endothelium 266

Vasculature 1968

Vinblastine 1998

Vinorelbine 1403

Vitamin-A 977, 2004

Vitamin-C 977

Vitamin-D 171

Vitamin-E 977

VM mouse 285

V $\alpha 24+N K T$-cells 74

Vulval cancer 1551

Western blotting 1102

Wilms' tumour 1557

X-irradiation 752

ZD2767 764

ZD9331 446 